\title{
Tratamento cirúrgico de dor neuropática em membro inferior por ferimento de arma de fogo. Relato de caso*
}

\author{
Surgical treatment of lower limb neuropathic pain caused by firearm injury. Case report.
}

Leonardo Eizo Watanabe ${ }^{1}$, Mário Yoshihide Kuwae ${ }^{2}$

* Recebido do Ambulatório de Microcirurgia de Mão do Hospital das Clínicas de Goiás. Goiânia, GO.

\section{RESUMO}

JUSTIFICATIVA E OBJETIVOS: O tratamento da dor neuropática é difícil e muitos pacientes apresentam alívio parcial e incompleto da dor com o tratamento farmacológico. Em casos de dor neuropática de origem traumática deve ser considerada a possibilidade de tratamento com a exploração cirúrgica, pois existe a possibilidade da dor ser causada por compressão nervosa devida a fibrose de tecidos adjacentes ou espessamento de bainha. O objetivo deste estudo foi apresentar um caso de sucesso no tratamento cirúrgico de dor neuropática causada por espessamento da bainha no nervo ciático esquerdo causado por ferimento de arma de fogo.

RELATO DO CASO: Paciente do sexo masculino, alvejado por tiro de arma de fogo na região glútea esquerda, evoluiu com dor em queimação na região posterior da coxa esquerda, com irradiação para face posterior da coxa, perna e pé, que foi parcialmente controlada por tratamento farmacológico com oxicodona, gabapentina e amitriptilina. Com a persistência das dores, foi feita a exploração cirúrgica no local de entrada do projétil que evidenciou a presença de bulbo em nervo ciático esquerdo e espessamento da bainha, sendo feita endoneurólise para a descompressão de fibrose intraneural. Após a cirurgia fez uso de pregabalina, morfina e amitriptilina, e depois de 10 meses da cirurgia estava assintomático, sem uso das medicações.

1. Fisiatra do Ambulatório de Microcirurgia de Mão do Hospital das Clínicas de Goiás. Goiânia, GO, Brasil.

2. Ortopedista Responsável pelo Ambulatório de Microcirurgia de Mão do Hospital das Clínicas de Goiás. Goiânia, GO, Brasil.

Endereço para correspondência:

Dr. Leonardo Eizo Watanabe

Avenida Vereador Jose Monteiro, 1655 - Negrão de Lima

74653-230 Goiânia, GO.

Fone: (62) 3232-3232

E-mail: lewatanabe@hotmail.com
CONCLUSÃO: O tratamento cirúrgico com endoneurólise para a descompressão de fibrose intraneural no nervo ciático esquerdo associado ao tratamento com amitriptilina e pregabalina, controlou com sucesso a dor neuropática causada por ferimento de arma de fogo.

Descritores: Anticonvulsivantes, Antidepressivos tricíclicos, Dor, Intervenção cirúrgica, Neuropatia femoral.

\section{SUMMARY}

BACKGROUND AND OBJECTIVES: Neuropathic pain is difficult to treat and many patients have partial and incomplete pain relief with pharmacological treatment. When the neuropathic pain has traumatic origin one should consider the possibility of treating with surgical exploration because pain may be caused by nervous compression due to adjacent tissues fibrosis or sheath thickening. This study aimed at presenting a successful case of surgical treatment of neuropathic pain caused by sheath thickening of left sciatic nerve, caused by firearm injury.

CASE REPORT: Male patient, reached by firearm shot in the left gluteus region, who evolved with burning pain in the posterior left thigh region, with irradiation to the posterior thigh face, leg and foot, which was partially controlled with pharmacological treatment with oxycodone, gabapentin and amitriptyline. As pain persisted, surgical exploration was performed at the bullet entry site, which has evidenced the presence of left sciatic nerve bulb and sheath thickening, being performed endoneurolysis for intraneural fibrosis decompression. After surgery, patient was treated with pregabalin, morphine and amitriptyline and 10 months after surgery he was asymptomatic and no longer having medication.

CONCLUSION: Surgical treatment with endoneurolysis for intraneural fibrosis decompression in left sciatic nerve associated to treatment with amitriptyline and pregabalin has successfully controlled neuropathic pain caused by firearm injury. 
Keywords: Anticonvulsants, Antidepressants tricyclics, Femoral neuropathy, Pain, Surgical intervention.

\section{INTRODUÇÃO}

A dor neuropática (DN) surge como uma consequência direta de uma lesão ou doença afetando o sistema somatossensitivo, e afeta negativamente a qualidade de vida dos pacientes, incluindo o funcionamento físico e emocional, além altos custos sociais ${ }^{1,2}$.

O tratamento da DN é difícil e muitos pacientes apresentam alívio parcial e incompleto da dor com o tratamento farmacológico ${ }^{3}$. Em casos de $\mathrm{DN}$ de origem traumática deve ser considerada a possibilidade de tratamento com a exploração cirúrgica, pois existe a possibilidade da dor ser causada por compressão nervosa devida a fibrose de tecidos adjacentes ou espessamento de bainha.

O objetivo deste estudo foi apresentar um caso de sucesso no tratamento cirúrgico de DN causada por espessamento da bainha em nervo ciático esquerdo causado por ferimento de arma de fogo.

\section{RELATO DO CASO}

Paciente do sexo masculino, 30 anos, alvejado por tiro de arma de fogo, sendo um com entrada na face póstero-lateral da coxa direita com saída pela região anterior e outro na região glútea esquerda.

Depois de 12 dias começou a apresentar dor em queimação na região posterior da coxa esquerda, com irradiação para face posterior da coxa, perna e pé. Exame radiológico da coxa esquerda mostrou a presença de projétil no terço proximal de coxa esquerda. (Figura 1). Foi prescrito anti-inflamatório não esteroide, porém não houve melhora.

A dor foi aumentando a intensidade até atingir 10, pela escala analógica visual (EAV). Fez uso de $10 \mathrm{mg}$ de oxicodona de liberação controlada a cada 12 horas e $1800 \mathrm{mg} /$ dia de gabapentina por 10 dias com persistência do quadro álgico. Foi associada $25 \mathrm{mg}$ /dia de amitriptilina, havendo acentuada diminuição da intensidade da dor com melhora importante do apetite e qualidade do sono a partir do segundo dia, porém evoluiu com xerostomia e constipação intestinal, sendo necessário enema para poder evacuar. Após 20 dias o paciente voltou ao consultório relatando queixa somente de queimação na planta do pé esquerdo principalmente ao deambular, sendo prescritos $30 \mathrm{mg}$ de morfina de liberação controlada a cada 12 horas em substituição à

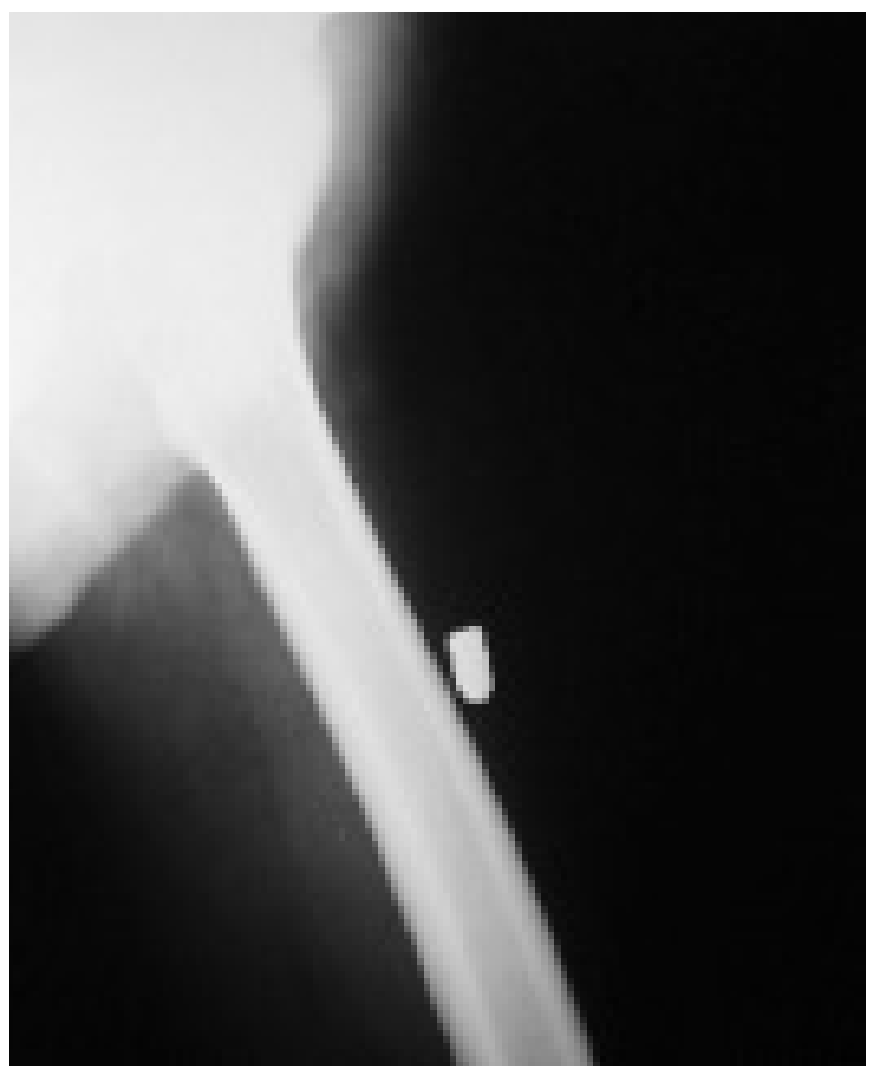

Figura 1 - Projétil de arma de fogo localizado no terço médio da coxa esquerda.

oxicodona. Após 15 dias o paciente retornou relatando que as dores persistiam, sendo indicada exploração cirúrgica no local de entrada do projétil na região glútea esquerda. A exploração cirúrgica evidenciou a presença de bulbo em nervo ciático esquerdo e espessamento da bainha, sendo feita endoneurólise para a descompressão de fibrose intraneural.

A gabapentina foi substituída por $75 \mathrm{mg}$ de pregabalina a cada 12 horas, e depois de 3 meses foi iniciada a redução gradativa da morfina e amitriptilina. Permaneceu com a pregabalina por mais 2 meses, e depois de 10 meses da operação estava assintomático, sem uso das medicações.

\section{DISCUSSÃO}

O tratamento da DN é desafiador, vários estudos clínicos aleatórios que investigaram o seu tratamento farmacológico, concluíram que não mais da metade dos pacientes experimentaram alívio significativo da dor, que geralmente é parcial ou incompleto. Por isso, na prática clínica, frequentemente são usados dois ou mais fármacos associados para se conseguir um efei- 
to benéfico aditivo e/ou redução dos efeitos adversos, principalmente se cada um deles atuar por diferentes mecanismos modulando os diferentes sistemas neurotransmissores ${ }^{2,3}$.

O tratamento farmacológico da $\mathrm{DN}$ deve considerar a eficácia clínica, os efeitos adversos, os efeitos sobre a qualidade de vida relacionada à saúde global, a conveniência e os custos. Os fármacos de primeira linha são os antidepressivos tricíclicos, os antidepressivos com inibição da recaptação de noradrenalina e serotonina, os ligantes alfa2-delta do canal de cálcio e a lidocaína tópica a 5\%. Os opioides, incluindo o tramadol, fármacos de segunda linha são apropriados para uso em determinados $\operatorname{casos}^{2-4}$.

O tratamento cirúrgico da DN pode ser feito em casos nos quais o tratamento farmacológico é ineficaz e a cirurgia pode ser benéfica para o paciente. Os procedimentos neurocirúrgicos habituais são modulativos com o uso da neuroestimulação ou de sistemas de implante de fármacos e ablativos com a lesão seletiva de alvos que mantêm os mecanismos da dor, mas também podem ser conservativos, quando é feita a neurólise para a descompressão de nervos ${ }^{5-9}$.

A neurólise quando indicada para o tratamento da DM de membros interiores geralmente diminui a intensidade da dor podendo mesmo tornar o paciente assintomático sem a necessidade do uso de medicamentos como no presente caso $^{8,9}$.

\section{CONCLUSÃO}

O tratamento cirúrgico com endoneurólise para a descompressão de fibrose intraneural em nervo ciático esquerdo associado ao tratamento com amitriptilina e pregabalina, controlou com sucesso a dor neuropática causada por ferimento de arma de fogo.

\section{REFERÊNCIAS}

1. Merskey H, Lindblom U, Mumford JM, et al. Pain terms. In: Merskey H, Bogduk N, (editors). Classification of chronic pain. Seattle: IASP Press; 1994. p. 207-13.

2. O'Connor AB. Neuropathic pain a review of the quality of life impact, costs, and cost-effectiveness of therapy. Pharmacoeconomics 2009;27(2):95-112

3. Dworkin RH, O'Connor AB, Backonja M, et al. Pharmacologic management of neuropathic pain: evidence-based recommendations. Pain 2007;132(3):237-51.

4. Dworkin RH, O'Connor AB, Audette J, et al. Recommendations for the pharmacological management of neuropathic pain: an overview and literature update. Mayo Clin Proc 2010;85(3 Suppl):S3-14.

5. Sindou MP, Mertens P, García-Larrea L. Surgical procedures for neuropathic pain. Neurosurgery Quarterly 2001;11(1):45-65.

6. Amid PK. A 1-stage surgical treatment for postherniorrhaphy neuropathic pain: triple neurectomy and proximal end implantation without mobilization of the cord. Arch Surg 2002;137(1):100-4.

7. Amid PK. Causes, prevention, and surgical treatment of postherniorrhaphy neuropathic inguinodynia: triple neurectomy with proximal end implantation. Hernia 2004;8(4):343-9.

8. Vora AM, Schon LC. Revision peripheral nerve surgery. Foot Ankle Clin 2004;9(2):305-18.

9. Schon LC, Anderson CD, Easley ME, et al. Surgical treatment of chronic lower extremity neuropathic pain. Clin Orthop Relat Res 2001;389:156-64.

Apresentado em 28 de setembro de 2010.

Aceito para publicação em 18 de maio de 2011. 